\title{
FIFTEEN YEARS OF CHANDRA OPERATION: SCIENTIFIC HIGHLIGHTS AND LESSONS LEARNED
}

Martin C. Weisskopf, NASA Marshall Space Flight Center, Astrophysics Office (ZP12)

SPIE 22 - 27 June 2014, Montréal, Quebec, Canada

Conference: AS102: Space Telescopes and Instrumentation 2014: Ultraviolet to Gamma Ray

NASA's Chandra X-Ray Observatory, designed for three years of operation with a goal of five years is now entering its 15-th year of operation. Thanks to its superb angular resolution, the Observatory continues to yield new and exciting results, many of which were totally unanticipated prior to launch. We will review some scientific highlights and present "lessons learned" from the experience of operating this great observatory. 